\title{
The perception and discrimination of local 3-D surface structure from deforming and disparate boundary contours
}

\author{
J. FARLEY NORMAN \\ Western Kentucky University, Bowling Green, Kentucky \\ and \\ SHANE R. RAINES \\ University of Pennsylvania School of Medicine, Philadelphia, Pennsylvania
}

\begin{abstract}
In a series of four experiments, we evaluated observers' abilities to perceive and discriminate ordinal depth relationships between separated local surface regions for objects depicted by static, deforming, and disparate boundary contours or silhouettes. Comparisons were also made between judgments made for silhouettes and for objects defined by surface texture, which permits judgment based on conventional static texture gradients, conventional stereopsis, and conventional structure-from-motion. In all the experiments, the observers were able to detect, with relatively high precision, ordinal depth relationships, an aspect of local three-dimensional (3-D) structure, from boundary contours or silhouettes. The results of the experiments clearly demonstrate that the static, disparate, and deforming boundary contours of solid objects are perceptually important optical sources of information about 3-D shape. Other factors that were found to affect performance were the amount of separation between the local surface regions, the proximity or closeness of the regions to the boundary contour itself, and for the conditions with deforming contours, the overall magnitude of the boundary deformation.
\end{abstract}

Occlusion boundary contours have long been known to contain a significant amount of information about an object's shape: For example, it is often easy to recognize both man-made and natural objects solely from their cast shadows or silhouettes (see Figure 1; see also Grafton, 1979; Hayward, 1998; Norman, Dawson, \& Raines, 2000). Indeed, before the invention of photography, silhouettes of prominent people were often made to record their portraits for posterity (Edouart, 1977). It is also apparent from the many petroglyphs and cave paintings that have been found around the world (e.g., Leakey, 1983; Sasse, 1996) that human observers have been creating recognizable silhouettes of objects and animals for many thousands of years. Leonardo da Vinci ( 1519/1970) hypothesized that "the first drawing was a simple line drawn 'round the shadow of a man cast by the sun on a wall" (p. 332). The perceptual utility of silhouettes and boundary contours seems obvious.

Given the research conducted over the past 70 years, we also know not only that silhouettes permit human observers to recognize and discriminate objects, but also that

Portions of the research described in this article were presented at the 1999 and 2000 annual meetings of the Association for Research in Vision and Ophthalmology. Two of the experiments (Experiments 2 and 3) were conducted in partial fulfillment of the requirements for the M.A. degree at Western Kentucky University (second author). Correspondence concerning this article should be addressed to J. F. Norman, Department of Psychology, Western Kentucky University, Bowling Green, KY 42101 (e-mail: farley.norman@wku.edu). when they deform over time, they allow us to perceive an object's shape and motion in three-dimensional (3-D) space and whether that motion is rigid or nonrigid (Cortese \& Andersen, 1991; Miles, 1931; Norman et al., 2000; Norman \& Todd, 1994; Pollick, 1994; Pollick, Nishida, Koike, \& Kawato, 1994; Todd, 1985; Wallach \& O' Connell, 1953). What we do not know, at the moment, is precisely what kind of information about shape human observers obtain when they view stationary or deforming silhouettes and boundary contours.

A variety of possibilities for deriving 3-D shape has emerged from the theoretical and mathematical study of boundary contours and silhouettes. One general possibility is that from boundary contours, human observers parse, or divide up, an object into distinct parts (Hoffman \& Richards, 1984; Koenderink, 1984a, 1984b, 1990; Koenderink \& van Doorn, 1976; Marr, 1982; Richards, Koenderink, \& Hoffman, 1987; Singh, Seyranian, \& Hoffman, 1999; Willats, 1992). For example, Willats proposed that we perceive objects defined by their silhouettes as being composed of "lumps" and "sticks." Koenderink and van Doorn (see also Koenderink, 1984a, 1984b, 1990) showed that the convexities and concavities of an object's boundary contour are the projections of areas of positive (i.e., "bump-like" surface regions) and negative (i.e., "saddlelike" surface regions) Gaussian curvature on the surface of the object, respectively. Thus, one can learn much about the intrinsic geometry of objects from the properties of their projected boundary contours. Richards et al. showed 


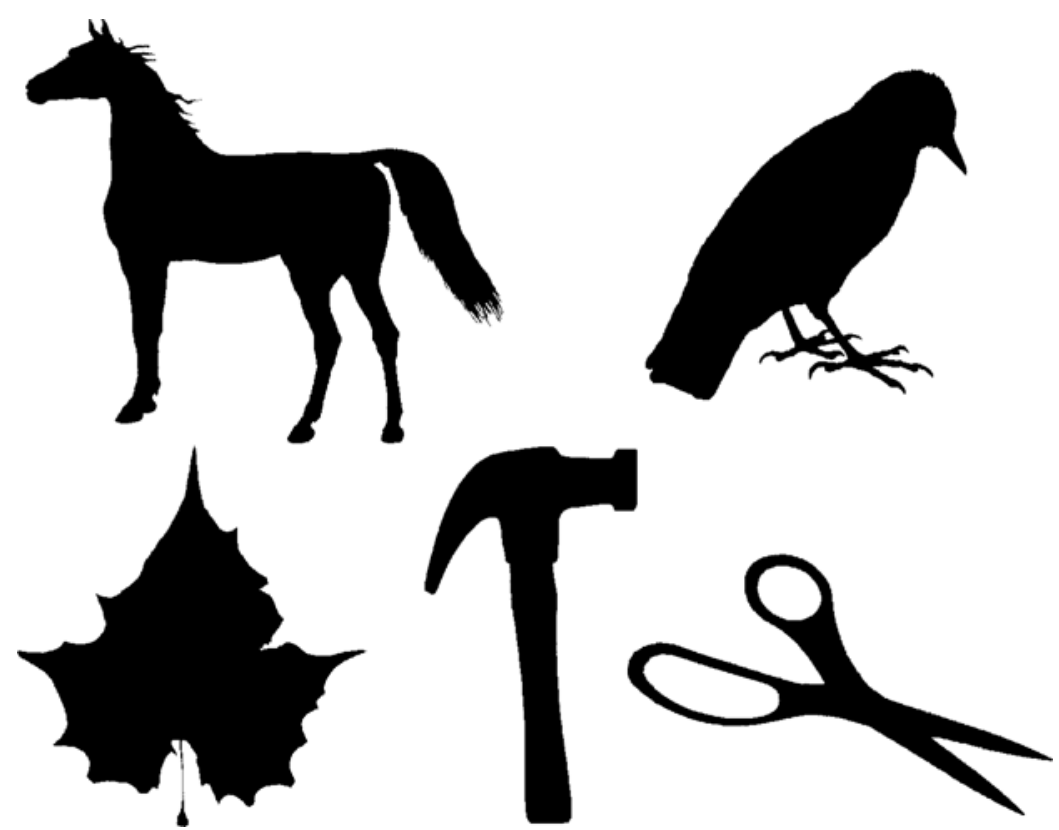

Figure 1, Silhouettes or cast shadows of various common animals and objects, both natural and man-made.

how this process can be extended in order to more uniquely interpret 3-D shape from boundary contours, using constraints such as those that involve the behavior of parabolic lines.

Another recent line of inquiry has demonstrated that at least within certain conditions, it is even possible to go beyond parts and determine local aspects of the 3-D structure of surface regions, such as their ordinal or metric depth, from static and deforming boundary contours. For example, Todd and Reichel (1989, see Figure 12) showed that within very small neighborhoods near the boundary, it is possible to determine ordinal depth relationships between adjacent regions. In addition, for dynamic situations in which objects and observers move relative to each other, it has also been shown that it is possible to obtain metric estimates of surface depth and/or curvature, particularly for areas near the boundary (Cipolla \& Blake, 1990; Giblin \& Weiss, 1987; Pollick, Giblin, Rycroft, \& Wilson, 1992). For these algorithms to work well, certain assumptions about the motion of the objects and observers need to be made. However, these analyses have shown that it is possible in principle for humans to determine local 3-D structure from the deforming boundary contours that occur when solid objects move relative to them. The purpose of the present set of experiments was to thoroughly investigate to what extent human observers can perceive and discriminate the differences in depth that occur between separated local regions on 3-D object surfaces that are defined only by their boundary contours. In the experiments, the perceptual informativeness of static, deforming, and disparate boundary contours was investigated. The distances (i.e., proximities) of the surface regions to the boundary were also manipulated to test how far the information provided by the occlusion boundary extends into the interior of an object's silhouette (cf. Tse, 2002).

\section{EXPERIMENT 1}

\section{Method}

Apparatus. The stimulus displays were rendered on a Power Macintosh 8600/300 using OpenGL and hardware acceleration (Nexus GA accelerator card, ATI Technologies, Inc.). The stimuli were displayed on a 21-in. Mitsubishi 91TXM monitor $(1,024 \times 768$ pixel screen resolution), using the perspective that was appropriate for the viewing distance from the observer to the monitor of $100 \mathrm{~cm}$. The stereoscopic displays were presented to the observers using CrystalEyes-2 Liquid Crystal Display (LCD) shuttered glasses (StereoGraphics, Inc.). The left and right stereoscopic images were alternately presented on the computer monitor at a rate of $150 \mathrm{~Hz}$. The LCD glasses operated in sync with the monitor, filtering the images so that the left stereoscopic half image was seen only by the left eye, whereas the right half image was seen only by the right eye. Each eye's view, then, was updated at a temporal rate of $75 \mathrm{~Hz}$.

Stimulus displays. One hundred smoothly curved objects composed of 2,048 connected, triangular polygons were generated randomly, following the procedures described by Norman and Todd (1996, 1998), Norman, Todd, and Phillips (1995), and Todd and Norman (1995). In brief, the object shapes were obtained by repeatedly modulating a sphere in depth with a unidimensional sinusoidal surface in an iterative fashion (10 iterations, the number of iterations controls the average complexity of the resulting objects). The orientation of the object was randomly changed in all three Cartesian coordinate axes between each iteration. The overall procedure resembled Fourier synthesis within a 3-D domain. The average diameter of the resulting 100 objects was $13.4 \mathrm{~cm}\left(7.67^{\circ}\right.$ of visual angle at the $100-\mathrm{cm}$ viewing distance). Four of these objects are shown in Figure 2. In this experiment, however, the objects were depicted without any image shading or texture and were visible only as solid blue 
silhouettes like those depicted in Figure 3. In this experiment, the informativeness of both static and deforming boundary contours was investigated.

Procedure. On any given trial, 1 of the 100 objects was randomly selected. In the conditions with motion, the object then rotated a full $360^{\circ}$ (in $5^{\circ}$ angular steps; the frame update rate was $15 \mathrm{~Hz}$ ) around a Cartesian vertical axis. As soon as the $360^{\circ}$ revolution was completed, the object stopped. At the same time, two surface regions were highlighted with small red spherical probe points $(0.125-\mathrm{cm}$ radius). The observers task was to indicate which of the regions underlying the probe points was closer to them in depth. No feedback about the observers' performance was given within a block of trials. The appearance of the probe points coincided exactly with the end of the movement, so that the probe points themselves never moved. In addition, in the stereoscopic conditions, the probe points were presented only to one eye's view. Since the probe points were always stationary and were presented monocularly, the only information the observers had on which to base their judgments of ordinal depth was the perception of shape generated from the disparate and/or deforming boundary contours presented during each trial (in addition, the computer's $z$-buffer was turned off when rendering the probe points; because of this, the shape of the spherical probe points in the projected image was always circular and independent of the surface structure of the object at the highlighted surface location; the probe points themselves, as rendered, contained no information that could influence the observers' judgments of ordinal depth). While the object was moving (i.e., prior to the appearance of the probe points), a single location on the objects' surface was highlighted to let the observer know in advance in which general area the probe points would appear later on, toward the end of the trial. It is important to note, however, that the movement of this single surface location itself carried no information about the shape of that general region; it merely served as a way to help direct the observers' attention so that they would not miss the appearance of the probe points at the end of the apparent motion sequence. If we had not directed the observers' attention toward a particular general region (remember that the projected size of the rendered objects was large, $141 \mathrm{~cm}^{2}$ ) prior to their judgments of ordinal depth, pilot testing showed that the observers would have had to abort a large percentage of trials during an experimental session, simply because they had not seen the probe points before the termination of a trial. The stimulus displays presented in the static boundary conditions were equivalent in every way to those used in the moving conditions; the only difference was the absence of motion/boundary deformation.

There was a total of 12 different experimental conditions formed by the orthogonal combination of three variables: 2 motion conditions (moving vs. static boundary contours) $\times 3$ surface separations (close, medium, and far; see Figure 4 for example separations) $\times 2$ disparity conditions (disparate boundary contours vs. no disparate boundary contours). It is possible to show binocularly disparate bound-
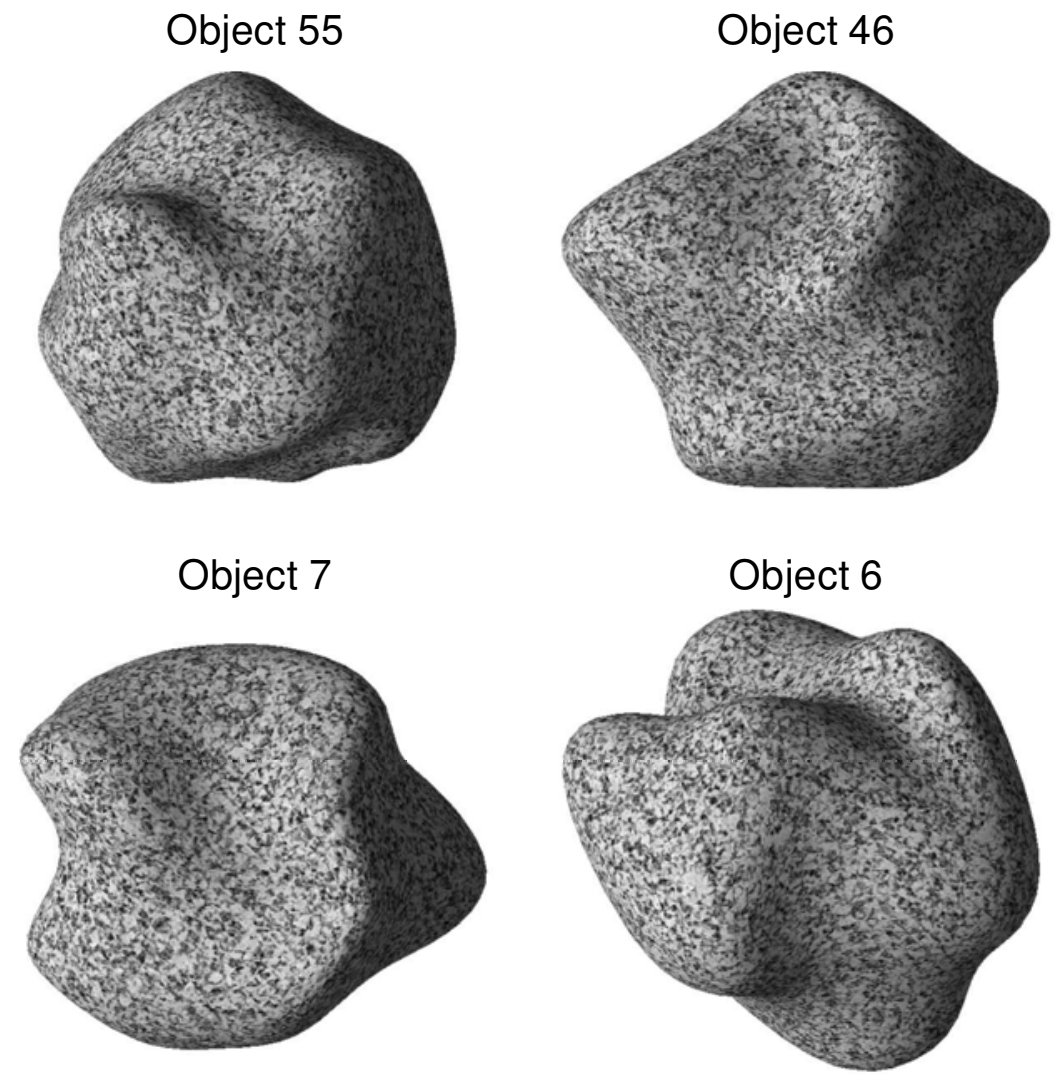

Figure 2. Four of the 100 objects used in Experiment 1, depicted with texture and lambertian shading. This figure also illustrates the variability in the complexity of the 100 object shapes used in the experiment. A measure of the complexity of object shape was operationally defined by the standard deviation of the distances of each vertex defining the object from the object's center (a completely smooth round object, such as a sphere, would thus have a complexity of zero). Object 55 was the least complex of all the objects, Object 6 was the most complex, whereas the complexity of Objects 7 and 46 was near the average for the entire set of objects. 

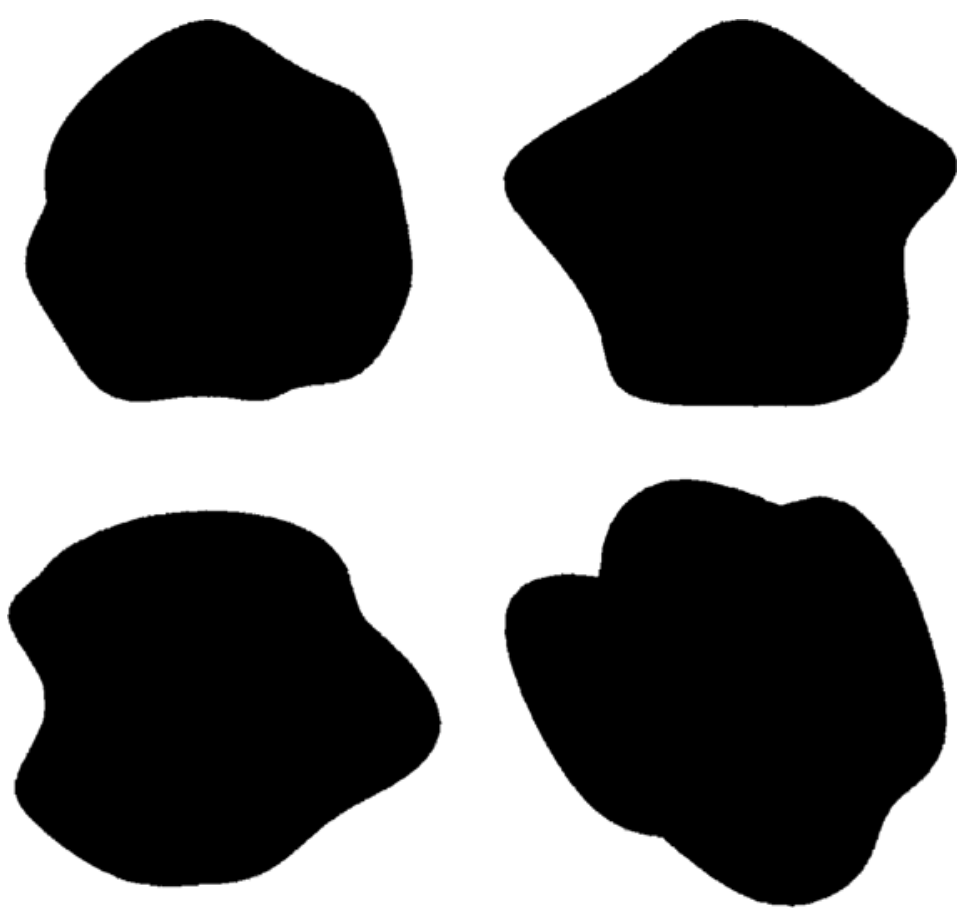

Figure 3. Silhouettes of the same four objects as those illustrated in Figure 2.

ary contours, since in ordinary binocular viewing, each eye sees a different boundary contour of the same 3-D object (i.e., the rim [Koenderink, 1990] is different for each eye). In the no-disparity conditions, the observers wore an eyepatch over one eye.

In each block, 50 trials were run at eight magnitudes of depth difference i.e., there were four depth differences where the right region was closer (by $0.125,0.375,0.625$, or $0.875 \mathrm{~cm}$ ) and 4 depth differences where the left region was closer (also by $0.125,0.375$, 0.625 , or $0.875 \mathrm{~cm}$ ). These eight particular depth differences were carefully chosen following pilot testing so that the same depth differences could be used in all the experimental conditions (variations in separation, static vs. moving boundary contours, etc.), and that would also allow for a reliable determination of thresholds in all cases. Each block contained 400 experimental trials (50 trials for eight depth differences) plus 20 practice trials. Two blocks were run for each of the 12 experimental conditions. A total of 9,600 trials was therefore performed by each of the 3 observers (12 conditions $\times 8$ depth differences $\times 100$ trials/depth difference/condition).

At this juncture, it is important to point out that our observers could not perform the ordinal depth discrimination task merely by picking the probe point closest to the center of the silhouette (this alternative strategy would be successful for pairs of regions on the surface of a sphere). On any given trial, a pair of points having a particular depth difference and separation was randomly chosen from 500 possible pairs of surface regions that were randomly culled from all possible pairs on the 100 randomly generated solid objects $(500 \times 8$ depth differences $\times 3$ surface separations $=12,000$ possible pairs of surface regions). For half of these pairs, the probe point closer to the center of the silhouette was indeed closest to the observer in depth; for the remaining half of the pairs, the probe point closer to the center of the silhouette was farthest from the observer in depth. Any observer who tried to use this alternative strategy would be unable to perform better than chance at the task. Performing this task accurately required the observers to estimate ordinal depth relationships per se from static, disparate, and deforming silhouette boundary contours.
Observers. The observers included the two authors (S.R.R. and J.F.N.), plus one additional experienced psychophysical observer (H.F.N.), who was naive regarding the specific purposes behind the experiment, was unfamiliar with how the stimuli had been generated, and so on. All had normal or corrected-to-no rmal visual acuity.

\section{Results and Discussion}

The psychometric functions (for the close and far separations) for observer J.F.N. in the moving condition with deforming boundary contours are shown in Figure 5. There is a large difference in the slopes of the psychometric functions, indicating a large effect of surface separation, so that discrimination performance is higher when the depths of nearby surface regions are compared than for more distantly separated regions (see also Norman \& Todd, 1996, 1998).

Ordinal depth discrimination thresholds were calculated by probit analysis, using a program developed by Foster and Bischof (1991). The 25th and 75th percentage points of the observers' psychometric functions (like those shown in Figure 5) indicated the thresholds for detecting when the right and the left surface regions were closer, respectively. The average ordinal depth discrimination thresholds (thresholds for detecting that the left region was closer and for detecting that the right region was closer were averaged) for all 12 conditions combined across all the observers are shown in Figure 6. One can clearly see the effect of separation on the surface and an extremely large effect of the motion. However, it is interesting that there was no effect of the disparate stereoscopic views [within-subjects analysis of variance (ANOVA), $F(1,22)=$ 
$0.306, p=.59]$. Consequently, the data for each observer were then collapsed across stereo/no-stereo.

The individual results (post-collapse) for each observer are shown in Figure 7. The effect of motion (i.e., deformation of boundary contour) was highly significant $[F(1,10)=111.8, p<.001]$, as was the effect of increasing surface separation $[F(2,10)=6.9, p=.013]$. The interaction was not significant; the presence of motion benefited performance equally at all surface separations: close, medium, and far.

\section{EXPERIMENT 2}

The results of Experiment 1 would seem to indicate that there is a large effect of deforming boundary contours but no effect of disparate boundary contours. All of the observers reported, however, that the stimulus displays were phenomenally much more "compelling" and "solid" in the stereoscopic conditions than in the nonstereoscopic conditions. Perhaps our observers' performance was not beneficially affected by the boundary contour disparity because it was not rendered at a sufficient resolution. The boundary contour disparity in our stimuli is small (see Figure 8). The main purpose of Experiment 2 was to examine how far inward the structural information provided by the boundary contour propagates toward the interior of the object. An important secondary purpose was to reinvestigate the role of disparate boundary contours, using higher resolution stimuli. Accordingly, the number of tri- angular polygons defining the solid objects was increased from 2,048 to 3,840 (an $87.5 \%$ increase), and the screen resolution of the frame buffer was increased from $1,024 \times$ 768 pixels to $1,280 \times 1,024$ pixels $(66.7 \%$ more pixels $)$.

\section{Method}

Apparatus. The apparatus was the same as that employed in Experiment 1, except that the stimulus displays were now accelerated by a Nexus 128 accelerator card (ATI Technologies, Inc.). Because of this change, the frame update rate for the apparent motion sequences was $20 \mathrm{~Hz}$.

Stimulus displays. The stimulus displays were identical to those used in Experiment 1, except that, in this experiment, the objects were defined by more polygons $(3,840)$ and were displayed at a higher screen resolution $(1,280 \times 1,024$ pixels $)$. Other than these differences, all other details of the stimulus construction were the same.

Procedure. On any given trial, 1 of the 100 possible objects was randomly selected. In the moving conditions, for two observers (S.R.R. and S.M.P.), the object oscillated back and forth over a $45^{\circ}$ range $\left( \pm 22.5^{\circ}\right)$ around a Cartesian vertical axis $\left(2.5^{\circ}\right.$ angular increment per frame transition). For the remaining observer (J.F.N.), the oscillation range was reduced to $24^{\circ}\left(3^{\circ}\right.$ angular increment per frame transition), since pilot observation showed that he required less boundary deformation for accurate discrimination performance. After three oscillations, the object's movement stopped. At the same time, two surface regions (with the same close separation as that used in Experiment 1) were highlighted with small red spherical probe points. The observers' task was to indicate which of the surface regions underlying the probe points was closer to them in depth. Once again, while the object was moving (i.e., prior to the appearance of the probe points), a single location on the object's surface
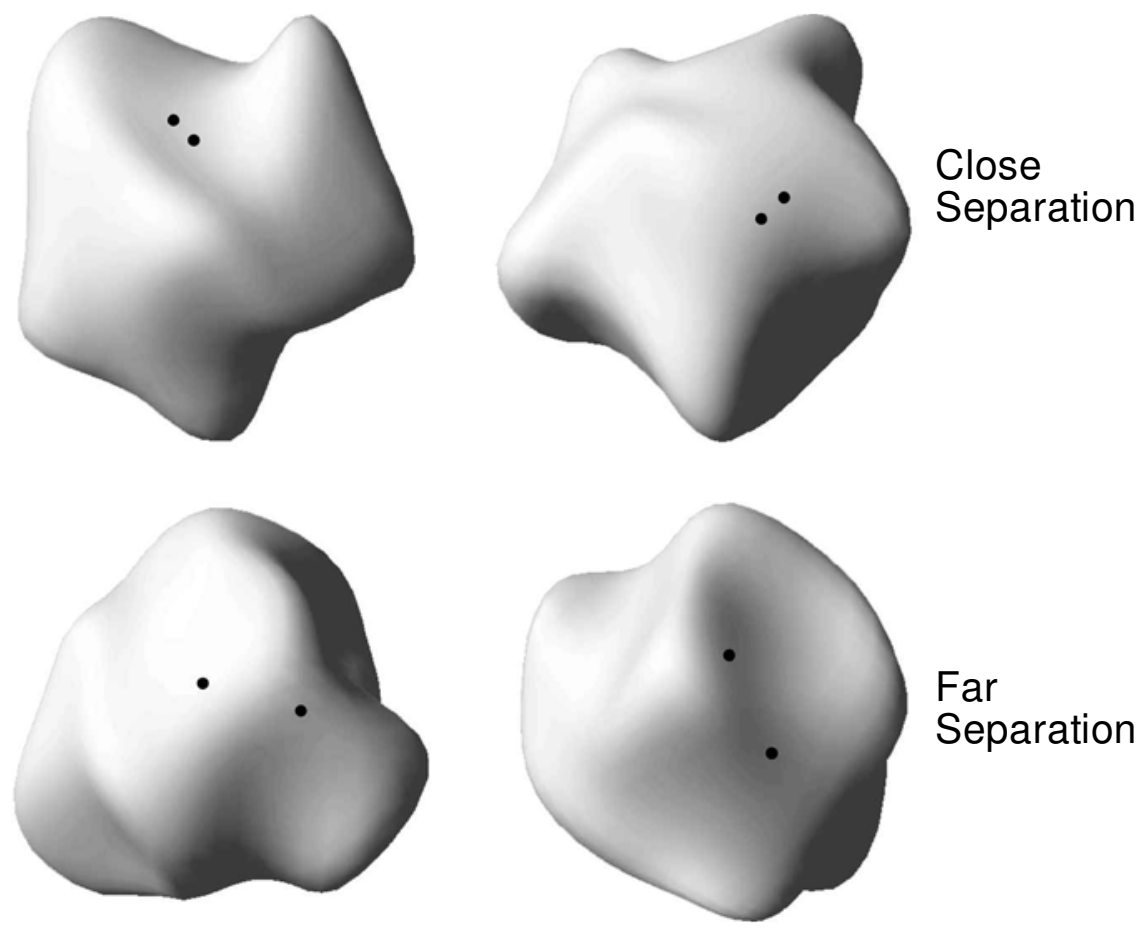

Figure 4. Examples of the close and far separation conditions used in Experiment 1. In this figure, the probe points highlighting the surface regions to be judged are larger (twice as large) than those actually used in the experiment. The objects in this figure are depicted with lambertian shading, whereas those used in the experiment were presented as silhouettes. 


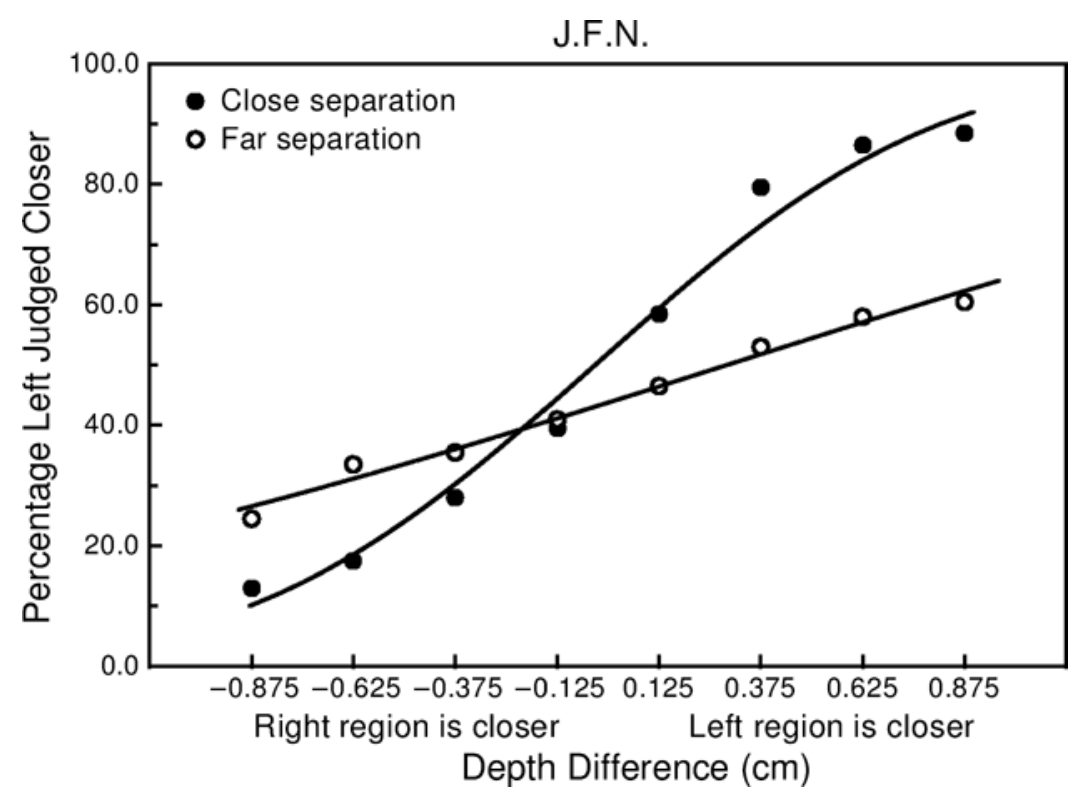

Figure 5. Psychometric functions for Observer J.F.N. in the deforming (i.e., moving) boundary contour condition for both close and far surface separations. The best-fitting cumulative normals have been fitted to the observer's data.

was highlighted to let the observer know in advance the general area in which the probe points would later appear at the end of the trial.

There was a total of 12 different experimental conditions formed by the orthogonal combination of three variables: 2 motion conditions (moving vs. static boundary contours) $\times 3$ distances of the pair of surface regions to be judged from the left or the right edges of the boundary contours (near, medium, and far; see Figure 9 for example distances from the boundary) $\times 2$ disparity conditions (disparate boundary contours vs. no disparate boundary contours). The dis- tances were measured relative to the left and the right edges of the boundary contours, since both the boundary disparity and the boundary deformation are concentrated there (since the objects rotated about a Cartesian vertical axis).

In each experimental block, 50 trials were run at six magnitudes of depth difference that is, there were three depth differences where the right region was closer (by $0.2,0.6$, and $1.0 \mathrm{~cm}$ ) and three depth differences where the left region was closer (also by $0.2,0.6$, and $1.0 \mathrm{~cm}$ ). Therefore, each block contained 300 experimental tri-

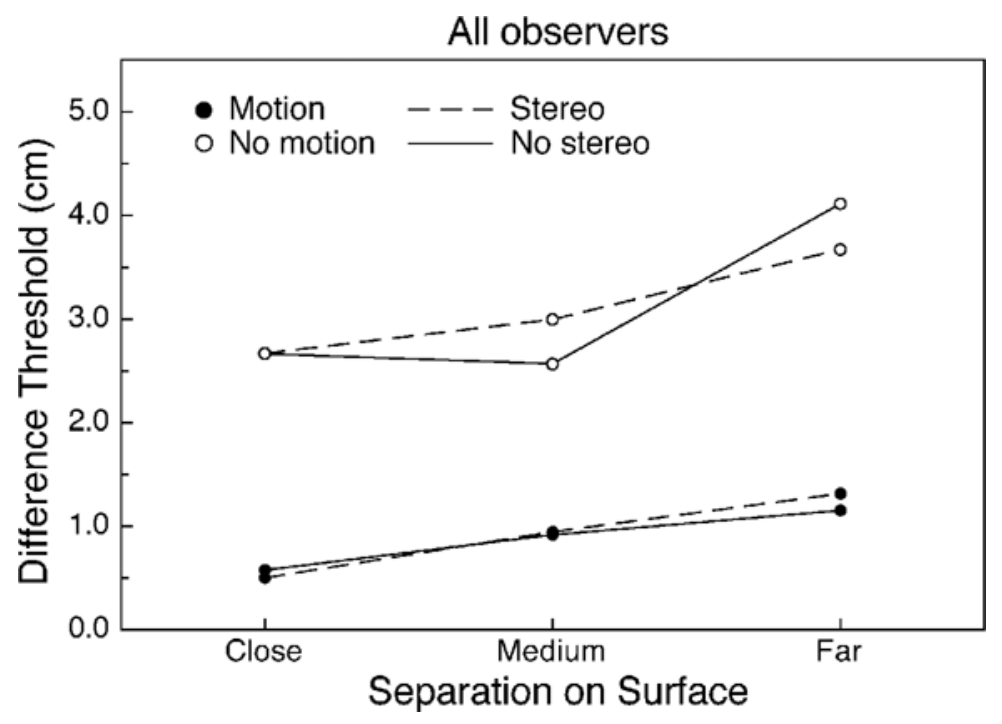

Figure 6. Overall results of Experiment 1 combined across all the observers, showing large effects of motion and surface separation but no effect of the presence or absence of stereoscopic views. 


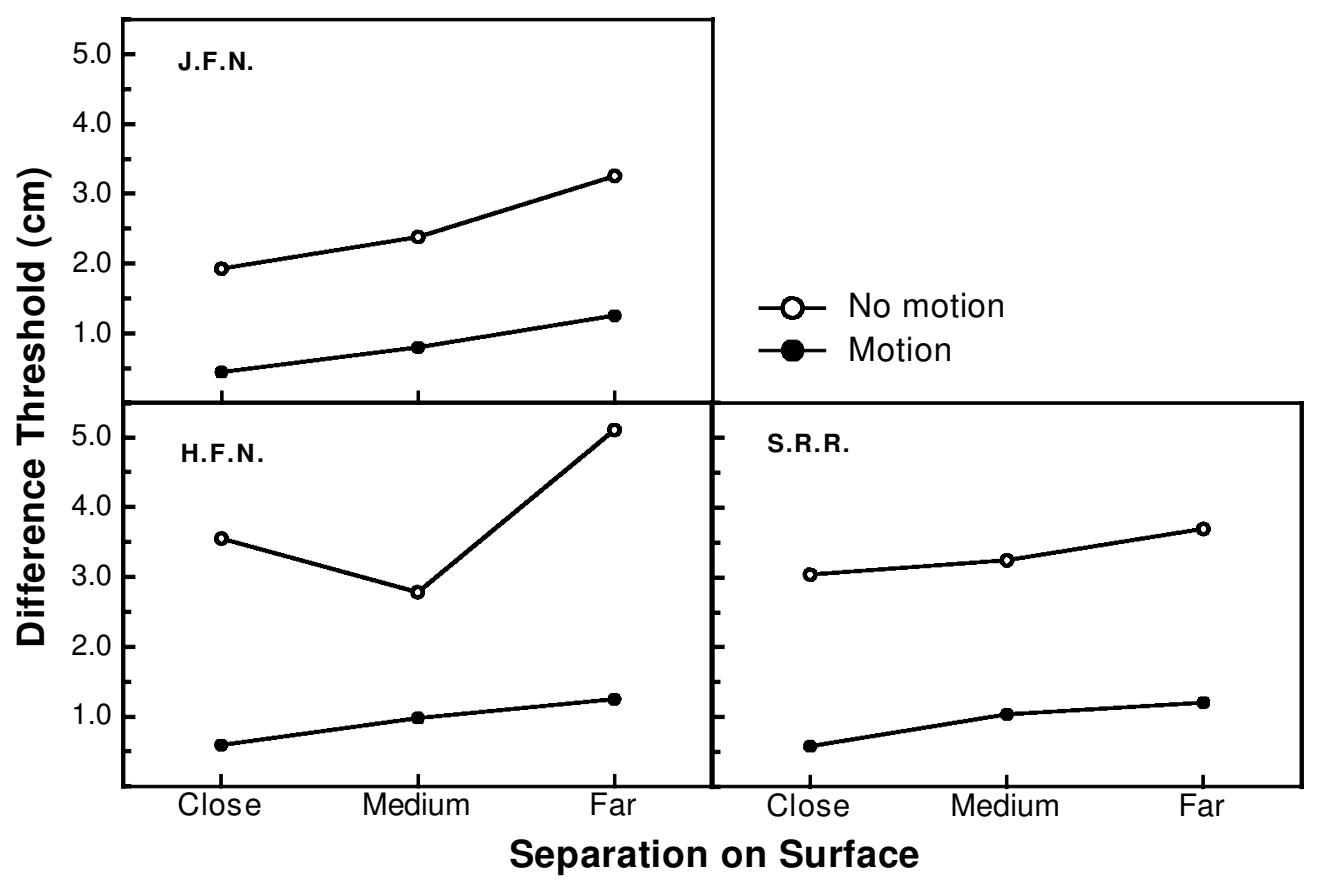

Figure 7. Results of the individual observers in Experiment 1 collapsed across the manipulation of stereoscopic views.

als plus 20 practice trials. Two blocks were run for each of the 12 experimental conditions. A total of 7,200 trials were performed by each of the 3 observers $(12$ conditions $\times 6$ depth differences $\times 100$ trials/depth-difference/ condition).
Observers. The observers included the two authors (J.F.N. and S.R.R.), plus one additional observer (S.M.P.), who was naive with regard to the exact purposes of the experiment. All observers had normal or corrected-to-normal visual acuity.

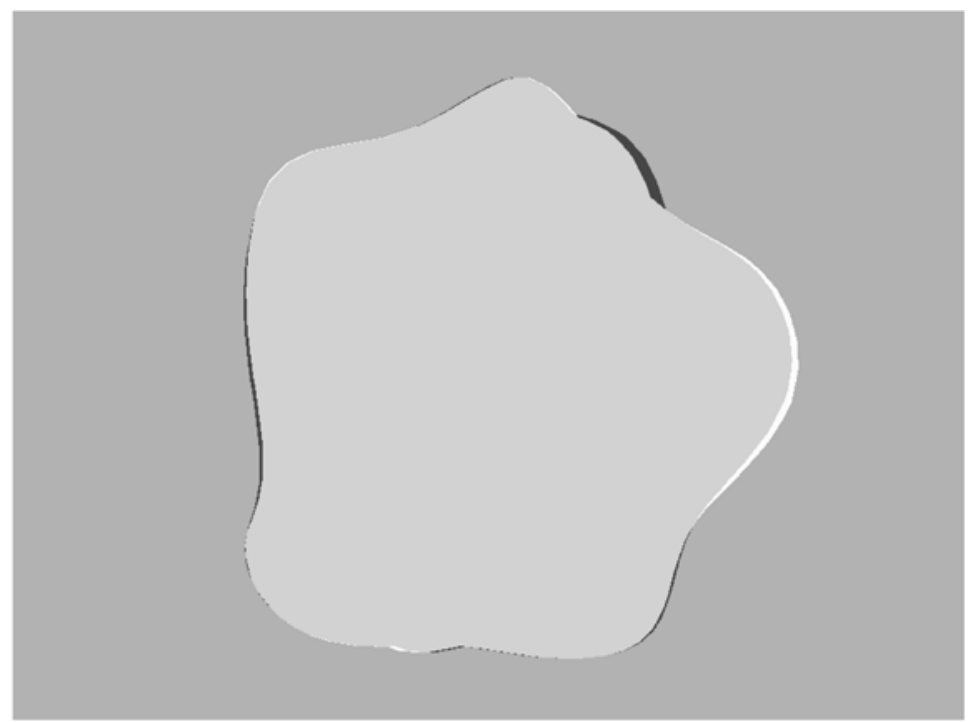

Figure 8. Schematic diagram illustrating the nature and magnitude of the stereoscopic boundary disparity for one of the objects used in Experiment 1 . The central gray area indicates that portion of the object visible to both eyes, whereas the thin black and white regions around the periphery indicate those surface regions visible to only the observer's left or right eye. The black and white shaded areas, therefore, illustrate the boundary disparity that occurs when observers view a solid object with both eyes. 

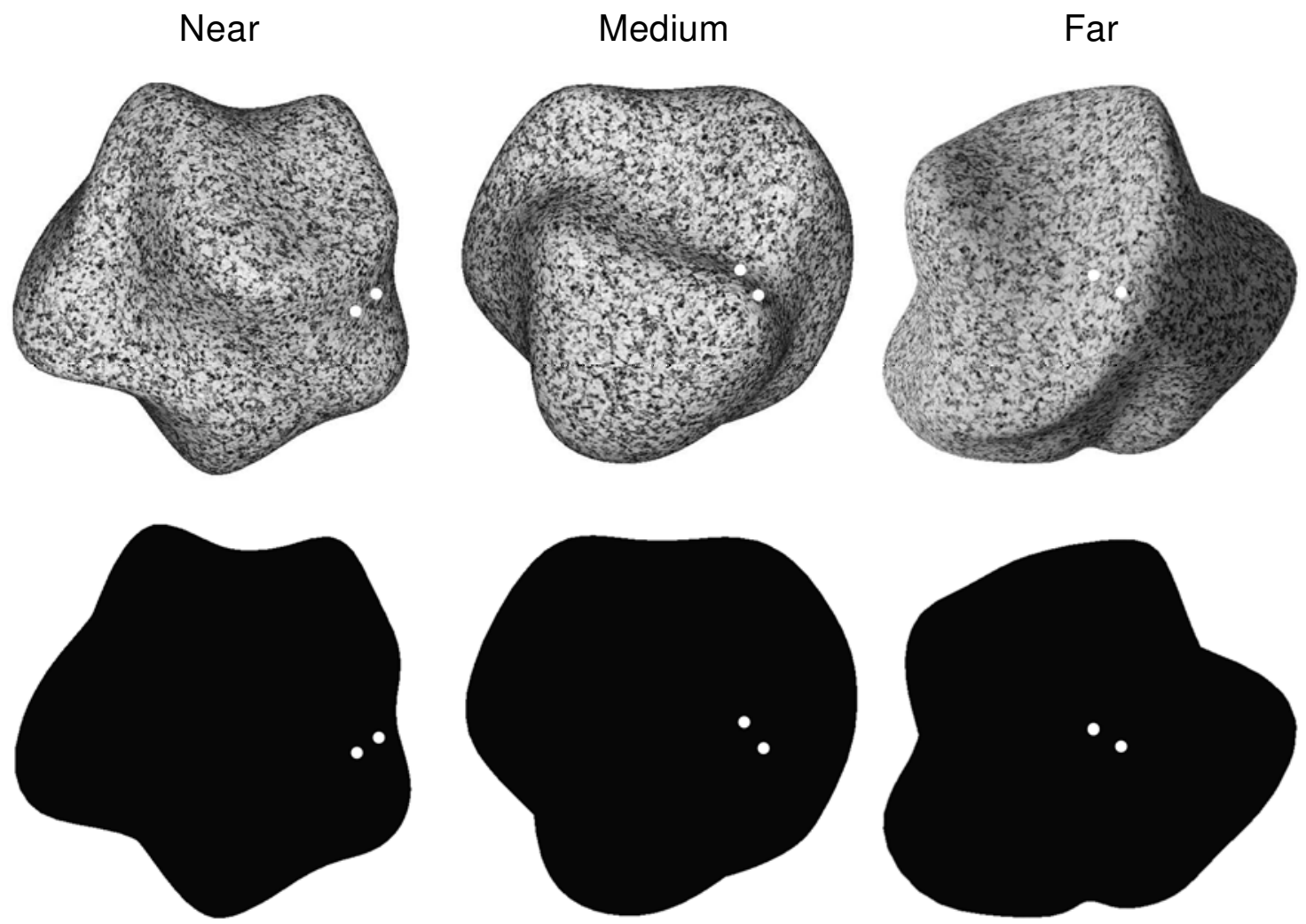

Figure 9. An illustration of the near-to-boundary, medium-distance-from-boundary, and far-from-boundary conditions used in Experiment 2 for three sample objects. The objects are depicted with texture and lambertian shading in the top row and as silhouettes in the bottom row. The probe points in this figure are larger than those used in the experiment.

\section{Results and Discussion}

Sample psychometric functions (near and far distances of the judged surface regions from the boundary)for observers S.R.R. and S.M.P. in the moving condition with binocularly disparate boundary contours are shown in Figure 10. One can see that there was some decrement for ordinal depth judgments when the regions to be compared were located at far distances from the boundary, but the decrement in many conditions was relatively small. It was still possible for the observers to make local ordinal depth judgments at well above chance levels for distances that were as far from the boundary as can occur for these objects. Information about ordinal depth provided from the moving and/or disparate boundary contours evidently does propagate to inner regions far removed from the boundary itself. The thresholds derived from all such psychometric functions were calculated by probit analysis and are shown in Figures 11 and 12, for observers S.M.P., S.R.R., and J.F.N., respectively.

The ordinal depth discrimination thresholds for all 12 conditions were subjected to a three-way within-subjects ANOVA. There was a main effect of the presence or absence of binocularly disparate boundary contours $[F(1,22)=$ $23.4, p<.001]$. There was also a main effect of moving boundary contours $[F(1,22)=45.7, p<.001]$. The effect of distance from the boundary was also significant $[F(2,22)=17.8, p<.001]$. In addition, there was a significant interaction between the presence of disparate boundary contours and the presence of moving boundary contours $[F(1,22)=10.3, p<.01]$. This interaction is clearly evident in the results obtained for Observers S.M.P. and S.R.R. (Figure 11). Notice from this figure that the presence of stereoscopic (i.e., disparate) boundary contours improved performance dramatically when no motion was present but did not markedly affect performance when motion was present. It would appear that the effects of disparate and moving boundary contours are not additive, in general (see, however, the results of Observer J.F.N. for a somewhat different pattern of results). For 2 out of 3 observers, the presence of either moving or disparate boundary contours led to good performance (i.e., lower thresholds), but having both present at the same time did not lead to further increases in performance.

\section{EXPERIMENT 3}

The first two experiments showed that deforming boundary contours lead to much better discrimination performance (i.e., lower thresholds) than do static boundary con- 


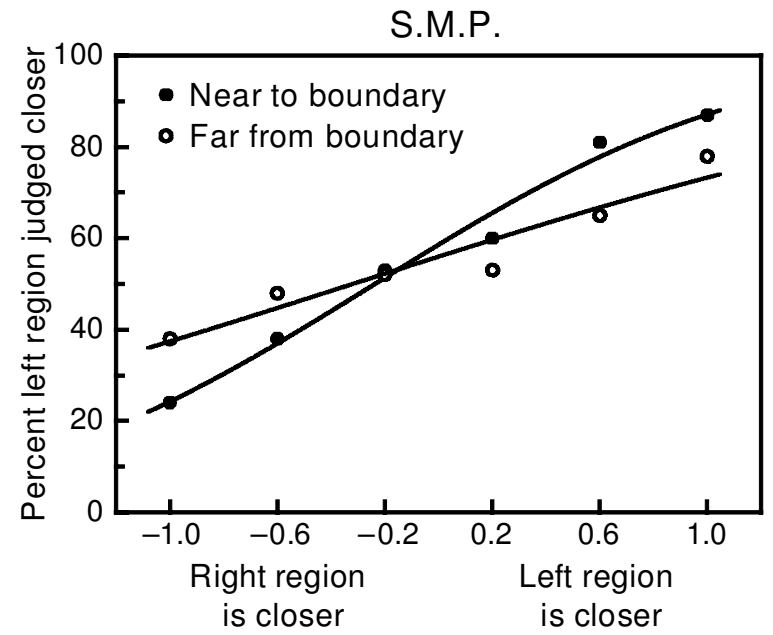

Depth Difference $(\mathrm{cm})$

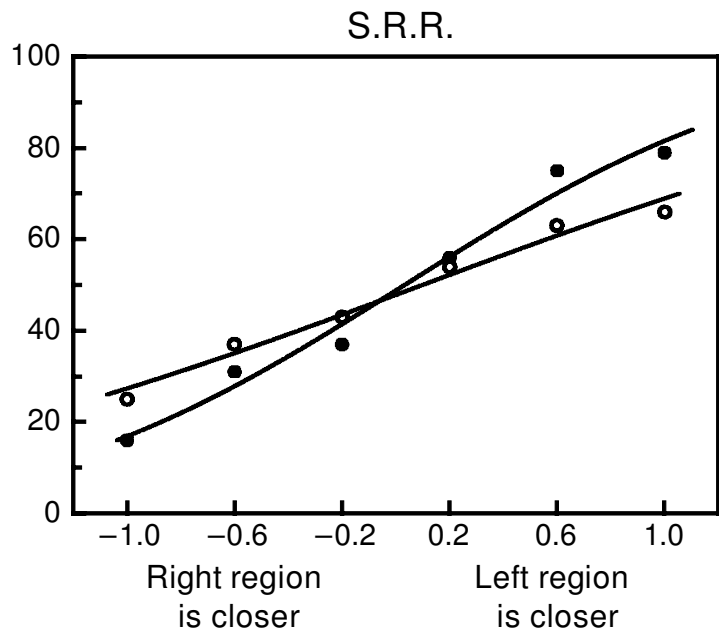

Depth Difference $(\mathrm{cm})$

Figure 10. Psychometric functions for Observers S.M.P. and S.R.R. in the combined stereo and moving condition for probe surface regions near to and far from the boundary. The best-fitting cumulative normals have been fitted to the observers' data.

tours. In particular, across both experiments, the thresholds for the motion conditions were $45.5 \%$ of those obtained in the otherwise equivalent no-motion conditions. The purpose of the third experiment was to examine the effects of the overall amount of boundary deformation upon the precision of the observers' ordinal depth judgments. In other words, how much boundary deformation is necessary for good perceptual performance?

\section{Method}

Apparatus. The apparatus was the same as that employed in Experiment 2 .

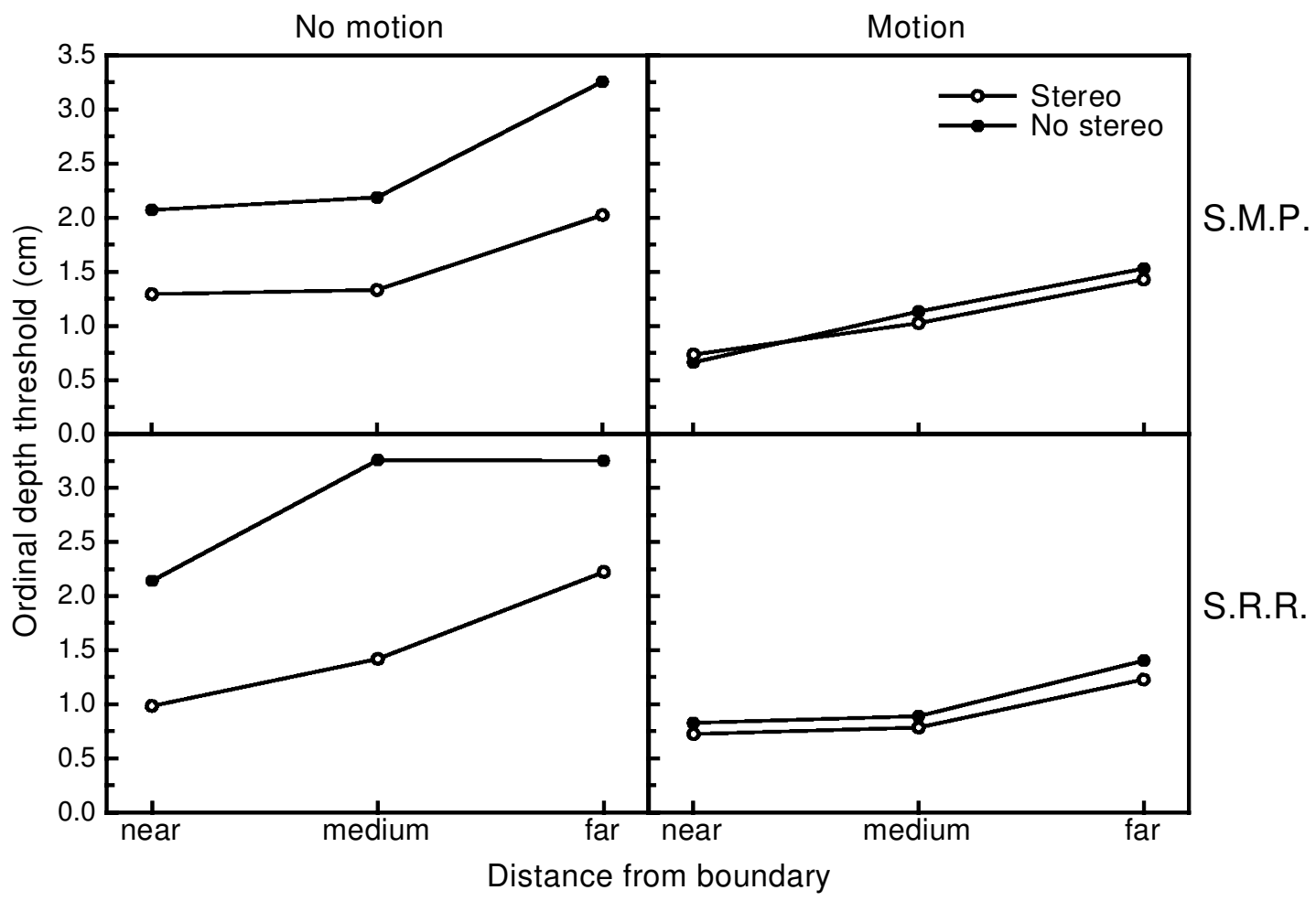

Figure 11. Results of Observers S.M.P. and S.R.R. for all the conditions employed in Experiment 2. 


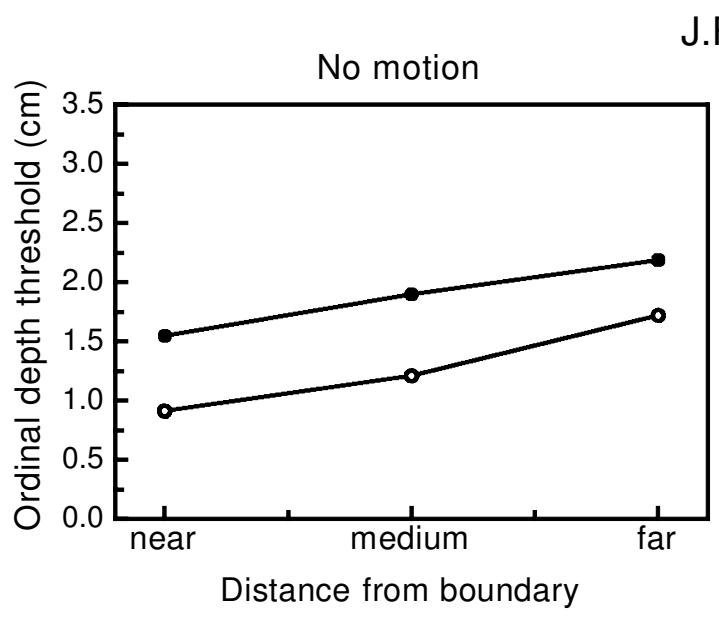

J.F.N.

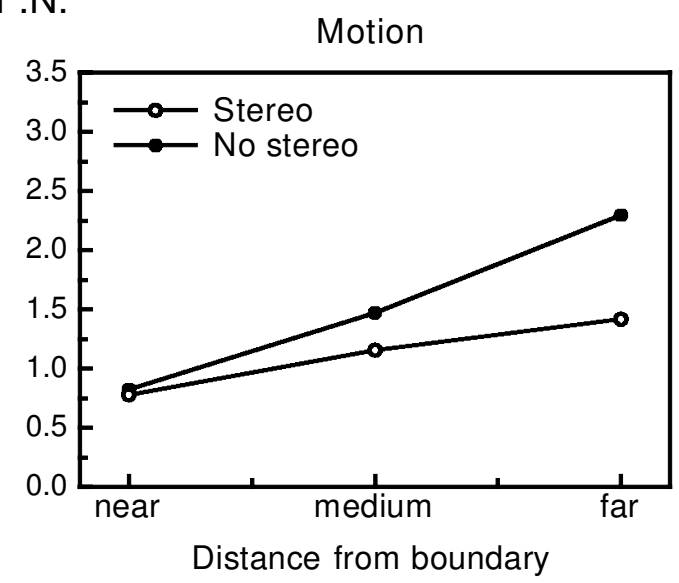

Figure 12. Results of Observer J.F.N. for all the conditions employed in Experiment 2.

Stimulus displays. All aspects of the stimulus displays were the same as those of the no boundary disparity conditions used in Experiment 2 .

Procedure. The experimental procedures and task were identical to those used in the near-to-boun dary condition of Experiment 2. In the present experiment, there were three different experimental conditions representing three different magnitudes of boundary deformation. The amount of boundary deformation was manipulated by varying the amount and range of object oscillation (i.e., the more rotation of the object in depth, the more boundary deformation that occurred). For Observers S.R.R. and H.F.N., the objects oscillated over ranges of $25^{\circ}, 35^{\circ}$, and $45^{\circ}$ (i.e., $\pm 12.5^{\circ}, \pm 17.5^{\circ}$, and $\pm 22.5^{\circ}$ ). Since Observer J.F.N. was more sensitive to boundary deformation, these ranges of oscillation were reduced to $12^{\circ}, 18^{\circ}$, and $24^{\circ}\left( \pm 6^{\circ}, \pm 9^{\circ}\right.$, and $\pm 12^{\circ}$ ).

In each block, 50 trials were run at six magnitudes of depth difference that is, there were three depth differences where the right region was closer (by $0.2,0.6$, or $1.0 \mathrm{~cm}$ ) and three depth differences where the left region was closer (also by $0.2,0.6$, or $1.0 \mathrm{~cm}$ ). Therefore, each block contained 300 experimental trials plus 20 practice trials. Two blocks were run for each of the three experimental conditions. A total of 1,800 trials were performed by each of the 3 observers $(3$ conditions $\times 6$ depth differences $\times 100$ trials/depth difference/condition).

Observers. The observers were the same as those who had participated in Experiment 1 (i.e., S.R.R., H.F.N., and J.F.N.).

\section{Results and Discussion}

The results are shown in Figure 13. The ordinal depth thresholds were calculated using probit analysis and are plotted in these figures as a function of the magnitude of boundary deformation (i.e., amount of object oscillation in depth). Notice that the thresholds for Observer J.F.N. are quantitatively very similar to those of Observers S.R.R. and H.F.N., despite the fact that J.F.N. viewed deforming boundary contours whose oscillation ranges were about half of those used for S.R.R. and H.F.N.. For those observers (J.F.N. and S.R.R.) who also participated in Experiment 2 , their thresholds for the no boundary deformation (i.e., static) condition are plotted for comparison purposes. It is readily apparent that even small amounts of boundary deformation are effective and lead to relatively good discrimination performance (for Observers J.F.N. and S.R.R., one can see a significant difference between the performances obtained in the lowest deformation condition of the present experiment and the analogous static condition of Experiment 2).

A within-subjects ANOVA for Observers S.R.R. and H.F.N. showed that their discrimination performances were significantly affected by the amount of boundary deformation, even though the effects on performance were relatively modest $[F(2,2)=48.1, p=.02]$. A Fisher LSD post hoc analysis showed that this significant main effect resulted from the improved performance in the $45^{\circ}$ oscillation condition relative to the $25^{\circ}$ and $35^{\circ}$ oscillation conditions. According to the post hoc analysis, there was no significant difference between the $25^{\circ}$ and $35^{\circ}$ oscillation conditions. An examination of J.F.N.'s results would tend to suggest a similar pattern: The $24^{\circ}$ range of object oscillation led to the best discrimination performance.

\section{EXPERIMENT 4}

The results of the previous three experiments have clearly demonstrated that static, disparate, and deforming boundary contours are effective sources of visual information about 3-D shape and can even lead to the perception of local 3-D surface structure. The purpose of this last experiment was to compare the performance obtained with static, disparate, and deforming boundary contours with that obtained with similar conditions when the objects also have visible surface texture. The presence of visible surface texture allows an observer to take advantage of other, more studied optical sources of information about 3-D shape, such as conventional binocular disparity (Howard \& Rogers, 1995; Julesz, 1971; Wheatstone, 1838), 3-D structure from motion (Braunstein, 1976; Braunstein \& Andersen, 1984; Norman \& Lappin, 1992; Todd, Akerstrom, Reichel, \& Hayes, 1988; Wallach \& O' Connell, 1953), and texture gradients (Gibson, 1950; Rosenholtz \& Malik, 1997; Todd \& Akerstrom, 1987). 

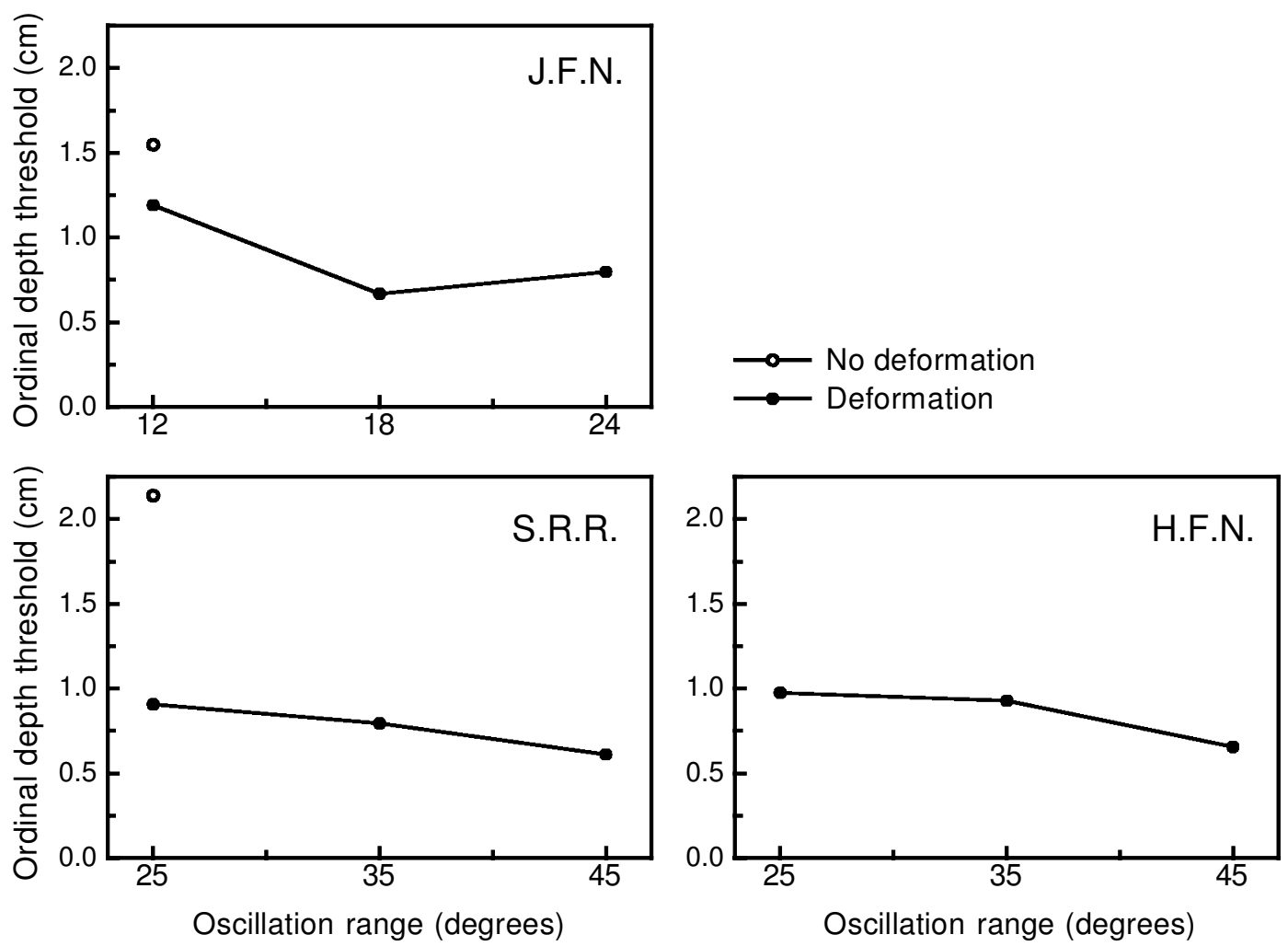

Figure 13. Results of Experiment 3 for Observers J.F.N., S.R.R., and H.F.N. (filled symbols). The performance for the analogous static boundary contour condition obtained in Experiment 2 for Observers J.F.N. and S.R.R. is also plotted for comparison (open symbols).

\section{Method}

Apparatus. The apparatus was the same as that employed in Experiments 2 and 3 .

Stimulus displays. All aspects of the stimulus displays were the same as those used in Experiments 2 and 3, except for the fact that in some conditions of the present experiment, the stimuli were not silhouettes but were rendered with a texture pattern resembling granite (as shown in Figures 2 and 9). Unlike those figures, however, the stimulus displays utilized in this experiment did not contain any image shading that is, texture and texture density gradients, but not shading, were present in the relevant conditions.

Procedure. The experimental procedures and task were identical to those of Experiments 2 and 3. The particular pairs of surface regions that were judged by the observers had the same close image separations as those used in Experiments 2 and 3 and were located near the boundary contour, like the surface regions used in Experiment 3. There was a total of eight different experimental conditions formed by the orthogonal combination of three variables: 2 motion conditions (moving vs. static boundary contours) $\times 2$ stereo conditions (stereo vs. no stereo) $\times 2$ texture conditions (texture vs. silhouette). In the moving conditions, the objects oscillated over a range of $45^{\circ}$ (Observers S.R.R. and H.F.N.) or $24^{\circ}$ (Observer J.F.N.) that is, the same values as those used in Experiment 2.

In each block, 50 trials were run at six magnitudes of depth difference that is, there were three depth differences where the right region was closer (by $0.2,0.6$, or $1.0 \mathrm{~cm}$ ) and three depth differences where the left region was closer (also by $0.2,0.6$, or $1.0 \mathrm{~cm}$ ). Therefore, each block contained 300 experimental trials plus 20 practice trials. Two blocks were run for each of the eight experimental conditions. A total of 4,800 trials were performed by each of the $3 \mathrm{ob}-$ servers $(8$ conditions $\times 6$ depth differences $\times 100$ trials/depthdifference/condition).

Observers. The observers were the same as those who had participated in Experiments 1 and 3 (i.e., S.R.R., H.F.N., and J.F.N.).

\section{Results and Discussion}

The results are shown in Figures 14-16. From an inspection of Figure 14, one can readily see the large and consistent effects of motion and texture for all 3 observers. There was an effect of motion for objects defined by texture and for objects depicted as silhouettes, although the overall effect of motion was greatest for silhouettes (especially for Observers S.R.R. and H.F.N.; see Figure 14). This motion $\times$ texture interaction was verified by a threeway within-subjects ANOVA $[F(1,14)=7.3, p<.05]$. The ANOVA also revealed significant main effects of motion and texture $[F(1,14)=48.6, p<.001$, and $F(1,14)=$ $124.5, p<.001$, respectively]. The proportional improvement in performance resulting from the movement and deformation of the boundary contour was just as large (Observer J.F.N.) or larger (Observers S.R.R. and H.F.N.) than the improvement that occurred when motion was added to textured surfaces. Thus, it is apparent from the results of this experiment that the deformation of an object's boundary contour is an important source of information for the human perception of even local aspects of object shape. 

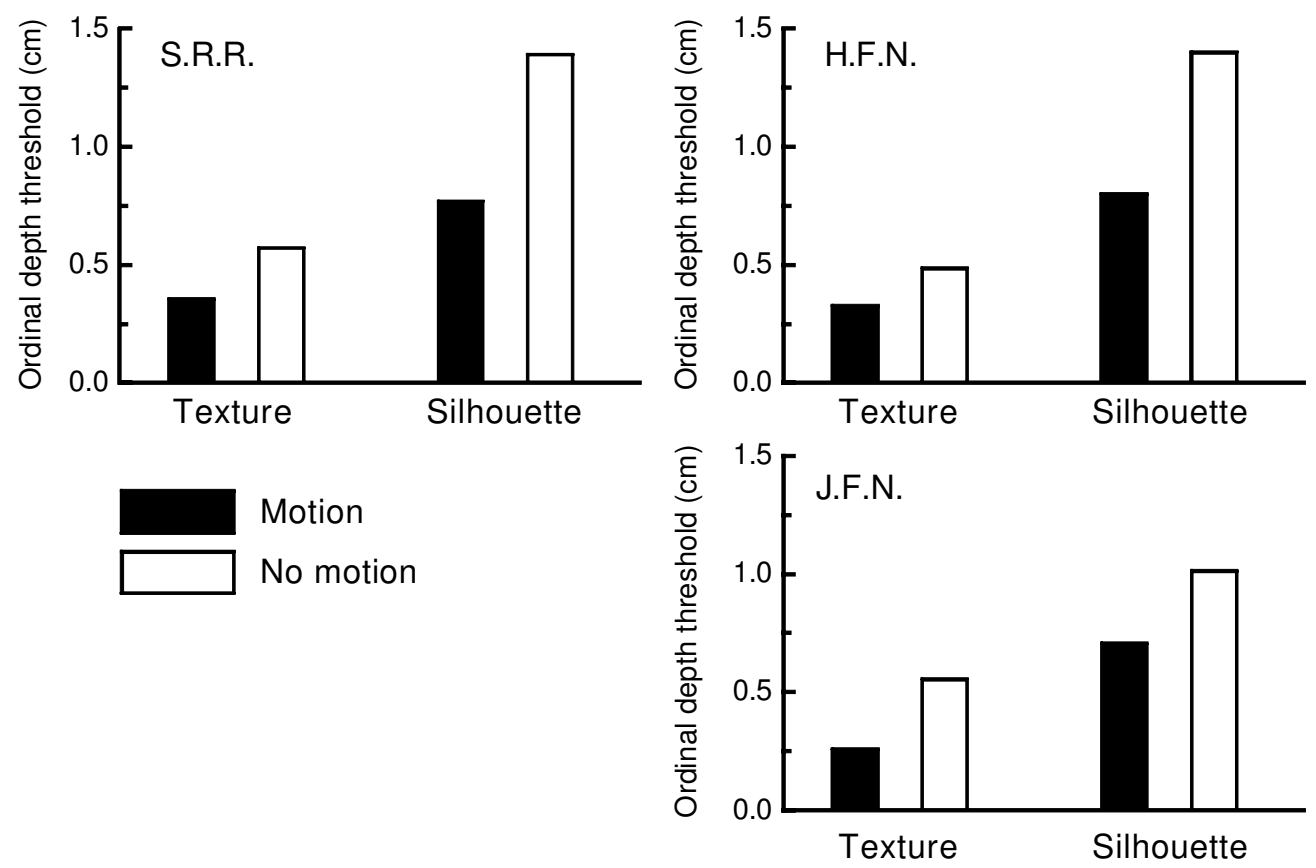

Figure 14. Results of Experiment 4, highlighting the effects of motion for objects presented both with and without texture (i.e., as silhouettes).

Figure 14 also shows that there is a rather significant effect for texture, even for statically presented objects (see the results depicted by the white bars). Indeed, within the no-motion, no-stereo conditions, the presence of static texture-density gradients led to an average decrease in threshold across observers of $51.3 \%$. This result suggests that static texture density gradients are a useful and important source of information about local shape, despite a long-held belief that they are relatively uninformative, as compared with other gradients, such as gradients of sizeshape (Phillips, 1970) and gradients of velocity (Braunstein, 1968; also see Braunstein, 1976, for an especially nice review of the literature concerning the informativeness of texture gradients). The large effect of texture density in the static no-motion, no-stereo conditions clearly indicates that texture is much more than a simple carrier for the velocities needed for perceived 3-D structurefrom-motion and for the binocular disparities needed for conventional stereoscopic vision.

Figure 15 shows the individual results for the manipulation of stereoscopic views both for objects defined by texture and for those depicted as silhouettes. There was a significant main effect of stereoscopic views $[F(1,14)=$ $36.6, p<.001]$, and the addition of stereoscopic views was just as beneficial for silhouettes as for objects defined by texture [i.e., no significant stereo $\times$ texture interaction; $F(1,14)=0.3, p>.05]$. It would appear, then, that disparate boundary contours provide human observers with a lot of information to support their perception of depth order; the effect of stereopsis is not limited to textured surfaces and conventional binocular disparity.
The results of the present experiment also showed that the presence of either stereoscopic views or motion aided performance but that the combination of stereoscopic views and motion did not further improve performance (i.e., the effects of stereo and motion do not add in an independent manner). This result can be seen in Figure 16 and was verified by a significant stereo $\times$ motion interaction $[F(1,14)=20.2, p<.001]$. The finding that stereoscopic disparities and motion both affect performance but that their effects do not add appreciably to produce even higher levels of performance when combined together has also been demonstrated for the perception of local surface orientation (Norman et al., 1995).

\section{GENERAL DISCUSSION}

Over the past 25 years, a number of mathematical analyses have shown that static and deforming boundary contours are a potentially rich source of information about the 3-D shape of solid objects. It has been shown, for example, that information about local surface depth (Cipolla \& Giblin, 2000; Giblin \& Weiss, 1987) and orientation (Barrow \& Tenenbaum, 1978; Brady, Ponce, Yuille, \& Asada, 1985; Brady \& Yuille, 1984) can be recovered from boundary contours. In addition, Koenderink and van Doorn (1976; see also Koenderink, 1984b; Richards et al., 1987) have demonstrated that the convexities and concavities of a boundary contour are informative about surface curvature. In particular, the convexities of a projected boundary contour indicate that the corresponding regions on the surface of an object have positive Gaussian curvature (i.e., are 

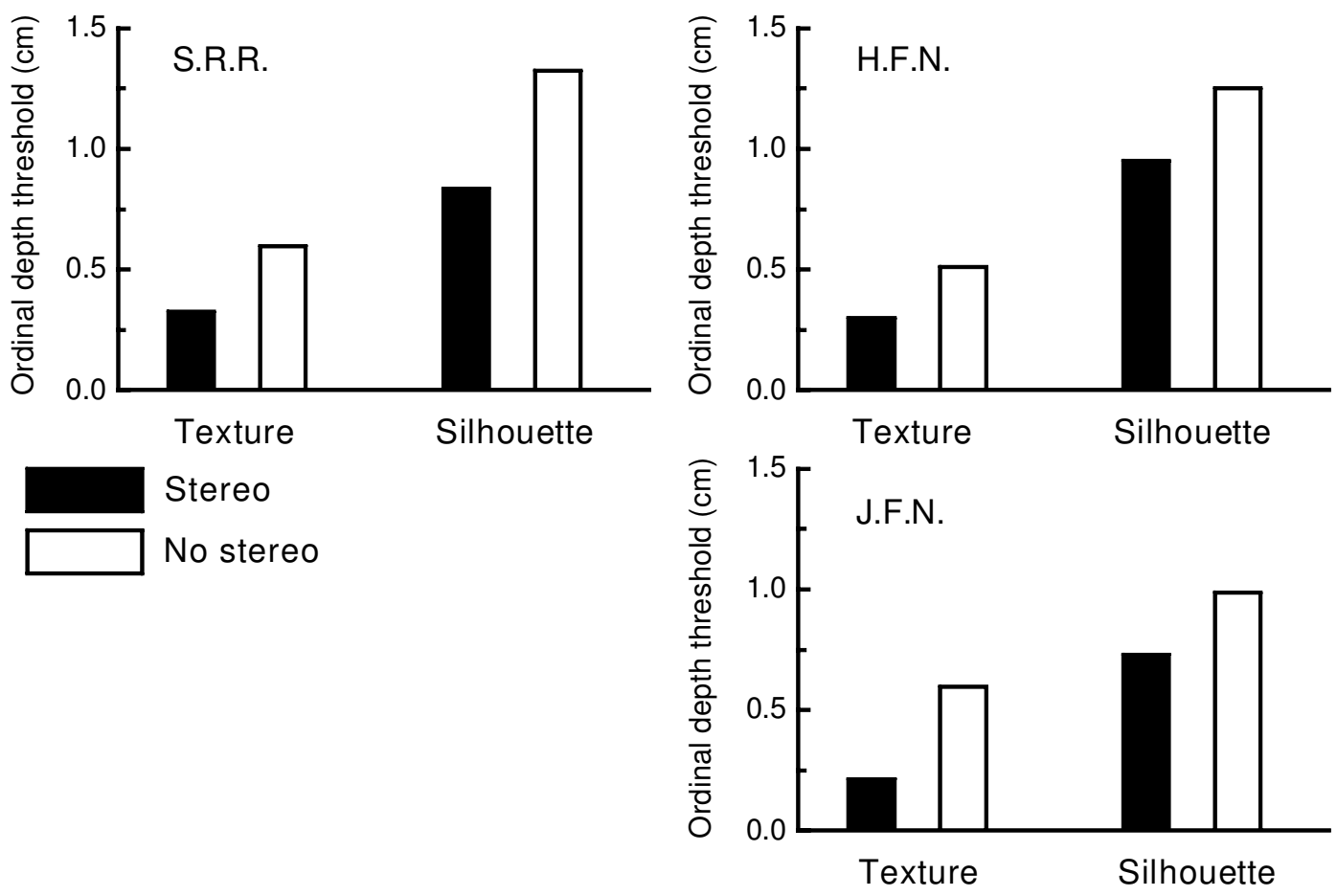

Figure 15. Results of Experiment 4, highlighting the effects of stereoscopic views for objects presented both with and without texture (i.e., as silhouettes).
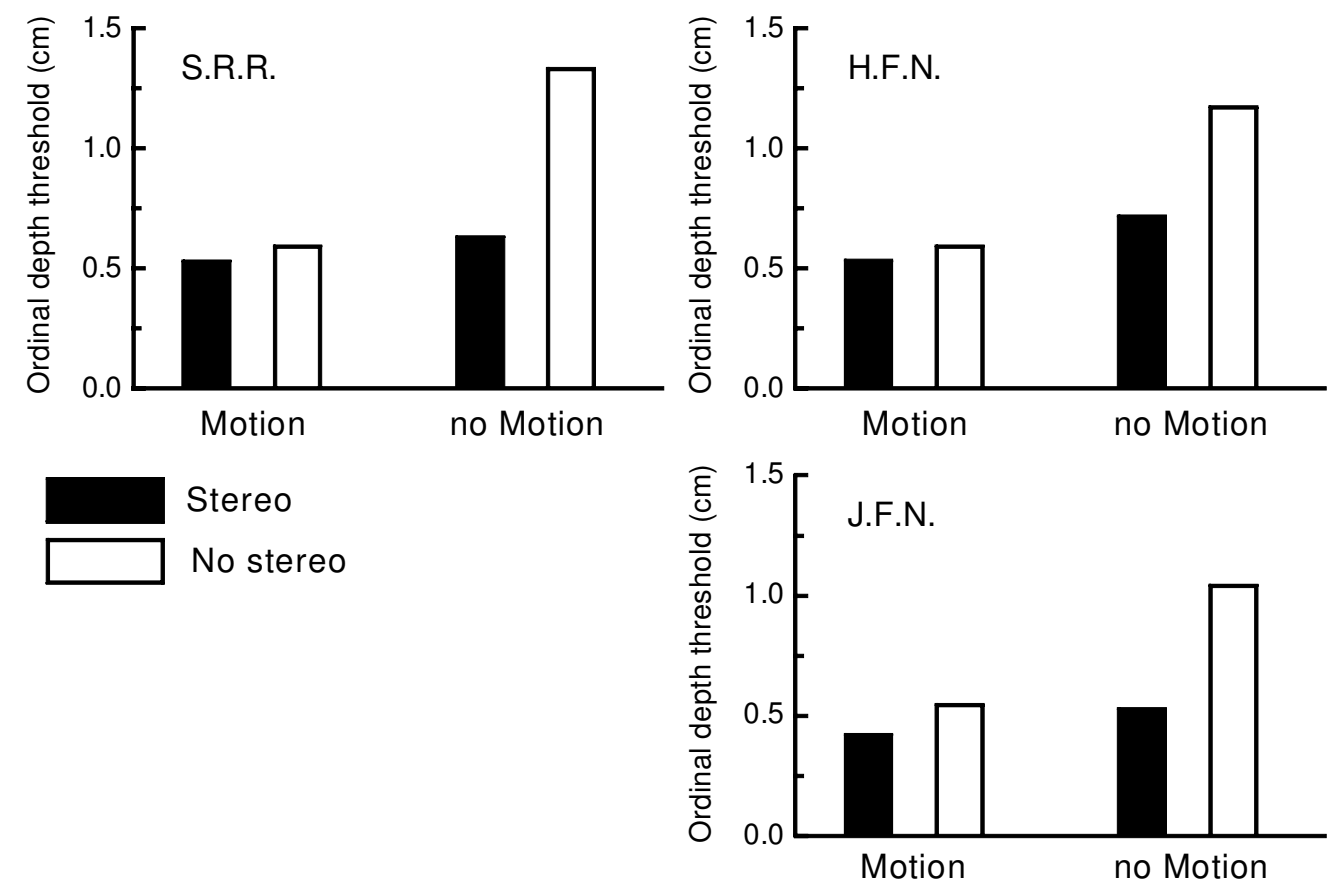

Figure 16. Results of Experiment 4, illustrating the effects of various combinations of stereoscopic views and motion. Note that the presence of stereoscopic views has a large effect when there is no motion; however, stereoscopic views do not improve performance when motion is present. 
"bump-like"), whereas concavities indicate that the corresponding surface areas have negative Gaussian curvature (i.e., are "saddle-like"). More recent research (Blake \& Cipolla, 1991; Cipolla \& Blake, 1992; see also Giblin \& Weiss, 1987) has shown that one can estimate local magnitudes of surface curvature from deforming boundary contours. Thus, as a whole, these mathematical analyses have shown that information about local surface depth, orientation, and curvature can potentially be obtained (at least if their computational assumptions are satisfied) from an object's stationary and deforming boundary contours.

Past perceptual research on the informativeness of boundary contours, using human observers, has focused on how accurately people can recognize objects from static and deforming boundary contours (Attneave, 1954; Hayward, 1998; Norman et al., 2000), how well they can estimate objects' global orientation in space (Oomes \& Dijkstra, 2002), and how they perceive their overall global shape (Cortese \& Andersen, 1991; Norman, Phillips, \& Ross, 2001) or motion in 3-D space (Norman \& Todd, 1994; Todd, 1985). The results of the present experiments extend this previous research by showing that human observers, in addition, can reliably estimate local aspects of 3-D surface structure (i.e., ordinal depth relationships between separated surface regions) from static, disparate, and deforming boundary contours. In addition, the present results demonstrate that from static, disparate, and deforming boundary contours, human observers obtain knowledge about the local 3-D structure of surface regions far removed from the boundary and its immediate vicinity (see Figures 10-12). Thus, the structural information provided by the boundary contour "propagates" toward the interior of the object (see also Tse, 2002).

The finding that disparate (Figures 11-12,15) and deforming boundary contours (Figures 7 and 14) both lead to significant improvements in the ability to detect ordinal depth relationshipsis an important one. As has been pointed out by Todd (1985), Cortese and Andersen (1991), and Norman and Todd (1994), the deformations of the boundary contour that accompany the rotation of a solid object in depth are quite different than the motions of projected texture elements that lead to the conventional kinetic depth effect (Wallach \& O' Connell, 1953) and, thus, cannot be analyzed by the same perceptual/physiological mechanisms. Likewise, the "disparity" that occurs between the boundary contours that are visible to an observer's left and right eyes is qualitatively different from conventional binocular disparities, a fact that was known and pointed out by Ogle (1950, see Figure 76, p. 139). In a situation characterizing conventional stereopsis and binocular disparity, the texture element or other feature that has the "disparity" or disparate position in the two eyes' views is the projection of the "same" location on the actual surface of the 3-D object. This correspondence does not exist for boundary contour disparity. This type of disparity is fundamentally different because "different locations" on the surface of the 3-D object are projecting to the boundary contour as seen by the observers' left and right eyes. Ogle (1950, p. 139) correctly describes this boundary contour disparity and concludes that "as a limiting condition, whole boundaries seen by one eye may be hidden to the other." Given that boundary contour disparity is quite distinct from conventional binocular disparity, it is an interesting and important result that it can facilitate, to a large degree, our perceptions of local 3-D surface structure. At the moment, we are unaware of any prior research that has unambiguously demonstrated that this distinctly different type of binoculardisparity influences and facilitates the perception of 3-D surface structure.

In the beginning of our investigation, the results that we obtained were something of a puzzle to us, given the prior mathematical analyses that had been performed showing the types of geometrical information that were theoretically present in boundary contours. Most of the prior analyses showed that boundary contours could deliver information about either surface orientation (e.g., Barrow \& Tenenbaum, 1978; Brady et al., 1985; Brady \& Yuille, 1984) or surface curvature (Blake \& Cipolla, 1991; Cipolla \& Blake, 1992; Giblin \& Weiss, 1987; Koenderink, 1984b; Koenderink \& van Doorn, 1976), but not depth. Even the analyses that could deliver information about surface depth (Giblin \& Weiss, 1987, p. 139; see also Cipolla \& Giblin, 2000, p. 85) obtained their estimates of depth secondarily following the recovery of the orientation of an object's tangent planes. Yet our preliminary pilot testing revealed that observers could not make accurate judgments about differences in either surface orientation or surface slant (a component of surface orientation; see Norman et al., 1995, and Stevens, 1983) from static and deforming boundary contours but that they were able to make relatively accurate judgments about differences in surface depth. At that time, our investigations seemed to show that human observers were equipped to perceive a different type of geometrical structure than the mathematical and computational analyses were able to recover from boundary contours. Todd and Reichel (1989) did discuss how information about ordinal depth could be derived from boundary contours, but their analysis applied only to surface regions very near an object's boundary contour, whereas our results indicated that observers can make ordinal depth judgments for surface regions located at relatively far distances from object boundary contours. Just recently, however (Mendonça, Wong, \& Cipolla, 2001; Wong \& Cipolla, 2001), new analyses have appeared that can apparently recover both observer motion and 3-D shape (surface depths and curvatures) from sequences of images of actual projected boundary contours of a moving object as recorded from a camera. It will be up to future research to investigate more precisely how human observers obtain knowledge about surface depth from static, disparate, and deforming boundary contours.

\section{REFERENCES}

Attneave, F. (1954). Some informational aspects of visual perception. Psychological Review, 61, 183-193.

Barrow, H. G., \& Tenenbaum, J. M. (1978). Recovering intrinsic scene characteristics from images. In A. R. Hanson \& E. M. Riseman (Eds.),

Computer vision systems (pp. 3-26). New York: Academic Press.

Blake, A., \& Cipolla, R. (1991). Robust estimation of surface curva- 
ture from deformation of apparent contours. Image \& Vision Computing, 9, 107-112.

Brady, M., Ponce, J., Yuille, A., \& Asada, H. (1985). Describing surfaces. Computer Vision, Graphics, \& Image Processing, 32, 1-28.

BRAdY, M., \& YuILle, A. (1984). An extremum principle for shape from contour. IEEE Transactions on Pattern Analysis \& Machine Intelligence, 6, 288-301.

Braunstein, M. L. (1968). Motion and texture as sources of slant information. Journal of Experimental Psychology, 78, 247-253.

Braunstein, M. L. (1976). Depth perception through motion. New York: Academic Press.

Braunstein, M. L., \& Andersen, G. J. (1984). Shape and depth perception from parallel projections of three-dimensional motion. Journal of Experimental Psychology: Human Perception \& Performance, 10, 749-760.

Cipolla, R., \& Blake, A. (1990). The dynamic analysis of apparent contours. In Proceedings of the Third IEEE International Conference on Computer Vision (pp. 616-623). Los Alamitos, CA: IEEE Computer Society Press.

Cipolla, R., \& Blake, A. (1992). Surface shape from the deformation of apparent contours. International Journal of Computer Vision, 9, 83-112.

Cipolla, R, \& Giblin, P. (2000). Visual motion of curves and surfaces. Cambridge: Cambridge University Press.

Cortese, J. M., \& Andersen, G. J. (1991). Recovery of 3-D shape from deforming contours. Perception \& Psychophysics, 49, 315-327.

EDOUART, A. A. C. F. (1977). Auguste Edouart's silhouettes of eminent Americans, 1839-1844. Charlottesville: University Press of Virginia.

FosteR, D. H., \& Bischof, W. F. (1991). Thresholds from psychometric functions: Superiority of bootstrap to incremental and probit variance estimators. Psychological Bulletin, 109, 152-159.

GIBLIN, P., \& WEISS, R (1987). Reconstruction of surfaces from profiles. In Proceedings of the First IEEE International Conference on Computer Vision (pp. 136-144).Los Alamitos, CA: IEEE Computer Society Press.

GiBson, J. J. (1950). The perception of the visual world. Boston: Houghton Mifflin.

GRAFTON, C. B. (1979). Silhouettes: A pictorial archive of varied illustrations. New York: Dover.

HAYWARD, W. G. (1998). Effects of outline shape in object recognition. Journal of Experimental Psychology: Human Perception \& Performance, 24, 427-440.

Hoffman, D. D., \& Richards, W. A. (1984). Parts of recognition. Cognition, 18, 65-96.

Howard, I. P., \& Rogers, B. J. (1995). Binocular vision and stereopsis. New York: Oxford University Press.

JuLESZ, B. (1971). Foundations of cyclopean perception. Chicago: University of Chicago Press.

KoEnderink, J. J. (1984a). The internal representation of solid shape and visual exploration. In L. Spillman \& B. R. Wooten (Eds.), Sensory experience, adaptation, and perception: Festschrift for Ivo Kohler (pp. 123-142). Hillsdale, NJ: Erlbaum.

KoenderinK, J. J. (1984b). What does the occluding contour tell us about solid shape? Perception, 13, 321-330.

Koenderink, J. J. (1990). Solid shape. Cambridge, MA: MIT Press.

Koenderink, J. J., \& VAn Doorn, A. J. (1976). The singularities of the visual mapping. Biological Cybernetics, 24, 51-59.

Leakey, M. D. (1983, July). Tanzania's stone age art. National Geographic, 164, pp. 84-99.

LEONARDO DA VINCI (1970). The practice of painting. In J. P. Richter (Ed.), The notebooks of Leonardo da Vinci (Vol. 1, pp. 241-332). New York: Dover. (Original work from 1519)

MARr, D. (1982). Vision. San Francisco: Freeman

Mendonça, P. R. S., Wong, K. K., \& Cipolla, R. (2001). Epipolar geometry from profiles under circular motion. IEEE Transactions on Pattern Analysis \& Machine Intelligence, 23, 604-616.

MiLES, W. R. (1931). Movement interpretations of the silhouette of a revolving fan. American Journal of Psychology, 43, 392-405.

Norman, J. F., Dawson, T. E., \& Raines, S. R. (2000). The perception and recognition of natural object shape from deforming and static shadows. Perception, 29, 135-148.
Norman, J. F., \& LAPPIn, J. S. (1992). The detection of surface curvatures defined by optical motion. Perception \& Psychophysics, 51, 386-396.

Norman, J. F., Phillips, F., \& Ross, H. E. (2001). Information concentration along the boundary contours of naturally shaped solid objects. Perception, 30, 1285-1294.

Norman, J. F., \& TodD, J. T. (1994). Perception of rigid motion in depth from the optical deformations of shadows and occlusion boundaries. Journal of Experimental Psychology: Human Perception \& Performance, 20, 343-356.

Norman, J. F., \& TodD, J. T. (1996). The discriminability of local surface structure. Perception, 25, 381-398.

Norman, J. F., \& TodD, J. T. (1998). Stereoscopic discrimination of interval and ordinal depth relations on smooth surfaces and in empty space. Perception, 27, 257-272.

Norman, J. F., Todd, J. T., \& Phillips, F. (1995). The perception of surface orientation from multiple sources of optical information. Perception \& Psychophysics, 57, 629-636.

Ogle, K. N. (1950). Researches in binocularvision. Philadelphia: Saunders.

Oomes, A. H. J., \& Dijkstra, T. M. H. (2002). Object pose: Perceiving 3-D shape as sticks and slabs. Perception \& Psychophysics, 64, 507-520.

Phillips, R J. (1970). Stationary visual texture and the estimation of slant angle. Quarterly Journal of Experimental Psychology, 22, 389-397.

Pollick, F. E. (1994). Perceiving shape from profiles. Perception \& Psychophysics, 55, 152-161.

Pollick, F. E., Giblin, P. J., Rycroft, J., \& Wilson, L. L. (1992). Human recovery of shape from profiles. Behaviormetrika, 19, 65-79.

Pollick, F. E., Nishida, S., Koike, Y., \& Kawato, M. (1994). Perceived motion in structure from motion: Pointing responses to the axis of rotation. Perception \& Psychophysics, 56, 91-109.

Richards, W. A., Koenderink, J. J., \& Hoffman, D. D. (1987). Inferring three-dimensional shapes from two-dimensional silhouettes. Journal of the Optical Society of America A, 4, 1168-1175.

Rosenholtz, R., \& MALIK, J. (1997). Surface orientation from texture: Isotropy or homogeneity (or both)? Vision Research, 37, 2283-2293.

SASSE, W. (1996). Prehistoric rock art in Korea: Pan' gudae. Korea Journal, 36, 75-91.

Singh, M., Seyranian, G. D., \& Hoffman, D. D. (1999). Parsing silhouettes: The short-cut rule. Perception \& Psychophysics, 61, 636-660.

Stevens, K. A. (1983). Slant-tilt: The visual encoding of surface orientation. Biological Cybernetics, 46, 183-195.

ToDD, J. T. (1985). Perception of structure from motion: Is projective correspondence of moving elements a necessary condition? Journal of Experimental Psychology: Human Perception \& Performance, 11, 689-710.

Todd, J. T., \& AKerstrom, R. A. (1987). Perception of three-dimensional form from patterns of optical texture. Journal of Experimental Psychology: Human Perception \& Performance, 13, 242-255.

Todd, J. T., Akerstrom, R. A., Reichel, F. D., \& Hayes, W. (1988). Apparent rotation in three-dimensional space: Effects of temporal, spatial, and structural factors. Perception \& Psychophysics, 43, 179-188.

Todd, J. T., \& Norman, J. F. (1995). The visual discrimination of relative surface orientation. Perception, 24, 855-866.

TodD, J. T., \& Reichel, F. D. (1989). Ordinal structure in the visual perception and cognition of smoothly curved surfaces. Psychological Review, 96, 643-657.

TSE, P. (2002). A contour propagation approach to surface filling-in and volume formation. Psychological Review, 109, 91-115.

Wallach, H., \& O'Connell, D. N. (1953). The kinetic depth effect. Journal of Experimental Psychology, 45, 205-217.

Wheatstone, C. (1838). Contributions to the physiology of vision: Part the first. On some remarkable, and hitherto unobserved, phenomena of binocular vision. Philosophical Transactions of the Royal Society of London, 128, 371-394.

Willats, J. (1992). Seeing lumps, sticks, and slabs in silhouettes. Perception, 21, 481-496.

Wong, K. K., \& Cipolla, R. (2001). Structure and motion from silhouettes. Proceedings of the 8th IEEE International Conference on Computer Vision, 2, 217-222. 